\title{
LATTICE VIBRATIONS WITH RAYLEIGH DISSIPATION
}

\author{
J. N. BOYD ${ }^{1}$ and P. N. RAYCHOWDHURY ${ }^{1}$
}

(Received 26 February 1997)

\begin{abstract}
We approximate a linear array of coupled harmonic oscillators as a symmetric circular array of identical masses and springs. The springs are taken to possess mass distributed along their lengths. We give a Lagrangian formulation of the problem of finding the natural frequencies of oscillation for the array. Damping terms are included by means of the Rayleigh dissipation function. A transformation to symmetry coordinates as determined by the group of rotations of the circle uncouples the equations of motion.
\end{abstract}

\section{Introduction}

Transformation to symmetry coordinates to separate coupled equations of motion for systems of harmonic oscillators is an elegant application of the representation theory of finite groups (see $[8,9]$ ). In the past, we have applied these methods to obtain the natural frequencies of vibration for various symmetric configurations of point particles interconnected with ideal springs $([1,2,4])$. We have also found applications for complex symmetry coordinates in other areas $([5,6])$.

In this paper, we introduce frictional, damping terms proportional to velocity into the equations of motion. By taking advantage of Rayleigh's dissipation function [7] to preserve the Lagrangian formulation of our problem, we are able to fit the computation of natural frequencies for underdamped systems into our previously developed scheme.

\section{Springs with extended mass}

Let us suppose that, in an array of particles connected by ideal springs, the $i$-th and $j$-th particles are connected by a spring of force constant $k$. Further, let us suppose

\footnotetext{
'Mathematical Sciences Department, Virginia Commonwealth University, Richmond, Virginia 23284-2014, USA.

(C) Australian Mathematical Society 2000 , Serial-fee code $0334-2700 / 00$
} 
that the mass $M$ of the spring is uniformly distributed along the length of the spring. We consider a motion of the two particles and spring in which all parts of the spring move parallel to its length.

We must compute the contribution of the moving spring to the total kinetic energy of the vibrating array. Let the undistorted length of the spring be $L$ and denote the displacements of the $i$-th and $j$-th particles with respect to their equilibrium locations by $x_{i}$ and $x_{j}$, respectively.

In Figure 1, the $i$-th particle is shown at the left. The positive directions of motion for both particles are to the right.

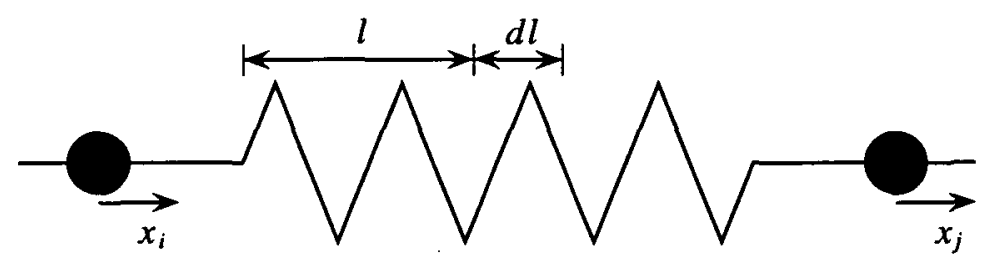

FIGURE 1. The spring with extended mass.

The length of the spring at any instant is given by $L+\left(x_{j}-x_{i}\right)$. We take the velocity of a bit of spring located at a distance $\ell$ to the right of the $i$-th particle to be

$$
\dot{x}_{i}+\frac{\ell}{L+\left(x_{j}-x_{i}\right)} \cdot\left(\dot{x}_{j}-\dot{x}_{i}\right) .
$$

The kinetic energy of this bit of the moving spring is

$$
d(K E)=\frac{1}{2} \frac{M}{L+\left(x_{j}-x_{i}\right)}\left(\dot{x}_{i}+\frac{\ell\left(\dot{x}_{j}-\dot{x}_{i}\right)}{L+\left(x_{j}-x_{i}\right)}\right)^{2} d \ell
$$

and the kinetic energy of the whole spring is

$$
\begin{aligned}
K E & =\frac{M}{2\left(L+\left(x_{j}-x_{i}\right)\right)} \int_{0}^{L+\left(x_{j}-x_{i}\right)}\left(\dot{x}_{i}+\frac{\ell\left(\dot{x}_{j}-\dot{x}_{i}\right)}{L+\left(x_{j}-x_{i}\right)}\right)^{2} d \ell \\
& =\frac{M}{6}\left(\dot{x}_{j}^{2}+\dot{x}_{j} \dot{x}_{i}+\dot{x}_{i}^{2}\right) .
\end{aligned}
$$

Thus we have obtained the contribution of a connecting spring to the overall kinetic energy of the array provided that the motions of all parts of the spring remain parallel to its own length. 


\section{Unitary transformations}

In the symmetric arrays which we shall consider, all motion will be confined to a fixed circle. As the number of particles $(N)$ and connecting springs grows very large, the circular arrangement may be taken to model a one-dimensional mechanical lattice of identical particles coupled with harmonic forces.

The symmetry groups for the arrays are the rotation groups $C(N)$ for $N$ identical point particles placed at the vertices of regular, plane $N$-sided polygons. The irreducible, unitary representations of $C(N)$ determine the unitary transformation matrix

$$
U=\frac{1}{\sqrt{N}}\left(u_{p, q}\right)
$$

where $u_{p, q}=\exp (2 \pi i p q / N)$ with $i^{2}=-1[2,3]$. We employ this matrix to accomplish the transformations to symmetry coordinates which separate the coupled equations of motion for small vibrations of our circular arrangements of particles and springs.

\section{Rayleigh's dissipation function}

To maintain the Lagrangian setting, we take advantage of Rayleigh's dissipation function which we denote by $\mathscr{F}$ [7]. We assume that the damping force acting on each particle is proportional to the particle's velocity. This damping force is not derivable from any potential energy but arises from frictional effects and is nonconservative. Its contribution to the total force acting on the $i$-th particle is $-\eta \dot{x}_{i}$, where the coefficient of the velocity has the same value for all particles and all directions of motion. We call $\eta$, which has a positive value, the damping coefficient.

In this case, Rayleigh's dissipation function is given by

$$
\mathscr{F}=\frac{1}{2} \eta \sum_{i=1}^{N} \dot{x}_{i}^{2}
$$

where the sum is taken over the $N$ particles in the array. The function may be interpreted in terms of the rate of energy loss by the array as it does work against friction. An increment in this work is

$$
d w=\sum_{i=1}^{N} \eta \dot{x}_{i} d x=\sum_{i=1}^{N} \eta \dot{x}_{i}^{2} d t .
$$

Thus $d w / d t=2 \mathscr{F}$ so that $\mathscr{F}$ must be half the rate of energy dissipation resulting from the action of friction upon the moving system. 
Since the frictional force upon the $i$-th particle may be obtained from $\mathscr{F}$ by partial differentiation $\left(-\partial F / \partial \dot{x}_{i}\right)$, the Lagrangian equation of motion for the array may be taken as

$$
\frac{d}{d t}\left(-\frac{\partial L}{\partial \dot{x}_{i}}\right)-\frac{\partial L}{\partial x_{i}}+\frac{\partial \mathscr{F}}{\partial \dot{x}_{i}}=0
$$

for $i=1,2,3, \ldots, N$.

\section{Example}

The computations for the frequencies of oscillation can be illustrated quite simply when the number of particles is small. Suppose that we have $N=3$ identical particles symmetrically arranged on a fixed circle as shown in Figure 2. The particles, which have mass $m$ and are connected to their neighbors by ideal springs, are constrained to move on the circle. The springs have force constant $k$ and possess uniformly distributed mass $M$. The damping coefficient for each particle is $\eta$.

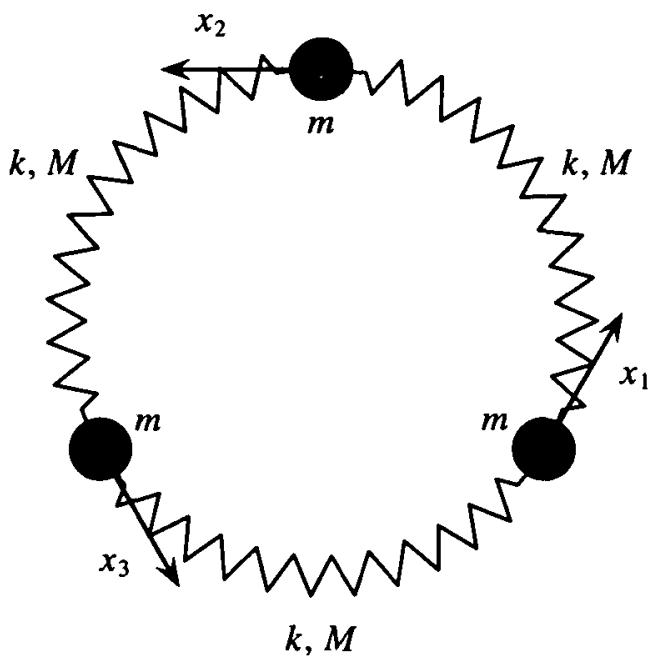

FIGURE 2. Three particles on a fixed circle.

We denote the tangential displacement from equilibrium of the $i$-th particle by $x_{i}$ and its velocity by $\dot{x}_{i}$. The positive sense of the motion is counterclockwise on the circle as indicated above.

The kinetic energy of the point masses is $m\left(\dot{x}_{1}^{2}+\dot{x}_{2}^{2}+\dot{x}_{3}^{2}\right) / 2$. Equation (1) implies that the kinetic energy associated with the extended masses of the springs is $M\left(2 \dot{x}_{1}^{2}+\right.$ 
$\left.2 \dot{x}_{2}^{2}+2 \dot{x}_{3}^{2}+\dot{x}_{1} \dot{x}_{2}+\dot{x}_{2} \dot{x}_{3}+\dot{x}_{3} \dot{x}_{1}\right) / 6$. The elastic potential energy contributed by the stretched or compressed springs is $k\left(2 x_{1}^{2}+2 x_{2}^{2}+2 x_{3}^{2}-2 x_{1} x_{2}-2 x_{2} x_{3}-2 x_{3} x_{1}\right) / 2$ and Rayleigh's dissipation function is $\eta\left(\dot{x}_{1}^{2}+\dot{x}_{2}^{2}+\dot{x}_{3}^{2}\right) / 2$.

Our goal is to write the equations of motion for the circular array in terms of separated symmetry coordinates. Having done that, we can easily list the frequencies of the natural modes of vibration. The computations can be stated simply and compactly in matrix notation. The correctness of the following matrix expressions can be easily verified.

The total kinetic energy may be written as

$$
\frac{1}{2}(m+M)\left(\dot{x}_{1} \dot{x}_{2} \dot{x}_{3}\right) I \operatorname{col}\left(\dot{x}_{1} \dot{x}_{2} \dot{x}_{3}\right)-\frac{1}{2}\left(\frac{M}{6}\right)\left(\dot{x}_{1} \dot{x}_{2} \dot{x}_{3}\right) V \operatorname{col}\left(\dot{x}_{1} \dot{x}_{2} \dot{x}_{3}\right),
$$

where $I$ is the $3-$ by-3 identity matrix and

$$
V=\left(\begin{array}{rrr}
2 & -1 & -1 \\
-1 & 2 & -1 \\
-1 & -1 & 2
\end{array}\right)
$$

- In passing, we note that $V$ is the matrix representation for the projection of the vectors of $\mathbb{R}^{3}$ onto a plane in which $x_{1}+x_{2}+x_{3}$ is constant [2].

The elastic potential energy is

$$
\frac{1}{2} k\left(x_{1} x_{2} x_{3}\right) V \operatorname{col}\left(x_{1} x_{2} x_{3}\right)
$$

and the dissipation function is

$$
\mathscr{F}=\frac{1}{2} \eta\left(\dot{x}_{1} \dot{x}_{2} \dot{x}_{3}\right) l \operatorname{col}\left(\dot{x}_{1} \dot{x}_{2} \dot{x}_{3}\right)
$$

The Lagrangian may now be written in the matrix form

$$
\begin{aligned}
L= & \frac{1}{2}(m+M)\left(\dot{x}_{1} \dot{x}_{2} \dot{x}_{3}\right) I \operatorname{col}\left(\dot{x}_{1} \dot{x}_{2} \dot{x}_{3}\right) \\
& -\frac{1}{2}\left(\frac{M}{6}\right)\left(\dot{x}_{1} \dot{x}_{2} \dot{x}_{3}\right) V \operatorname{col}\left(\dot{x}_{1} \dot{x}_{2} \dot{x}_{3}\right)-\frac{1}{2} k\left(x_{1} x_{2} x_{3}\right) V \operatorname{col}\left(x_{1} x_{2} x_{3}\right) .
\end{aligned}
$$

To obtain symmetry coordinates $z_{1}, z_{2}, z_{3}$ as in [2], we choose the transformation matrix for $N=3$,

$$
U=\frac{1}{\sqrt{3}}\left(\begin{array}{ccc}
-1 / 2+i \sqrt{3} / 2 & -1 / 2-i \sqrt{3} / 2 & 1 \\
-1 / 2-i \sqrt{3} / 2 & -1 / 2+i \sqrt{3} / 2 & 1 \\
1 & 1 & 1
\end{array}\right)
$$


which has inverse

$$
U^{-1}=\frac{1}{\sqrt{3}}\left(\begin{array}{ccc}
-1 / 2-i \sqrt{3} / 2 & -1 / 2+i \sqrt{3} / 2 & 1 \\
-1 / 2+i \sqrt{3} / 2 & -1 / 2-i \sqrt{3} / 2 & 1 \\
1 & 1 & 1
\end{array}\right) .
$$

The unitary matrix $U$ is generated by the irreducible representations of the group of plane rotations of the equilateral triangle. The transformation to complex symmetry coordinates is accomplished by taking the products

$$
\left(x_{1} x_{2} x_{3}\right) U^{1}=\left(\overline{z_{1} z_{2}} \bar{z}_{3}\right) \text { and } U \operatorname{col}\left(x_{1} x_{2} x_{3}\right)=\operatorname{col}\left(z_{1} z_{2} z_{3}\right),
$$

where $\overline{z_{i}}$ denotes the complex conjugate of $z_{i}$. The transformed Lagrangian is

$$
\begin{aligned}
L= & \frac{1}{2}(m+M)\left(\dot{x}_{1} \dot{x}_{2} \dot{x}_{3}\right) U^{-1}\left(U I U^{-1}\right) U \operatorname{col}\left(\dot{x}_{1} \dot{x}_{2} \dot{x}_{3}\right) \\
& -\frac{1}{2}\left(\frac{M}{6}\right)\left(\dot{x}_{1} \dot{x}_{2} \dot{x}_{3}\right) U^{-1}\left(U V U^{-1}\right) U \operatorname{col}\left(\dot{x}_{1} \dot{x}_{2} \dot{x}_{3}\right) \\
& -\frac{1}{2} k\left(x_{1} x_{2} x_{3}\right) U^{-1}\left(U V U^{-1}\right) U \operatorname{col}\left(x_{1} x_{2} x_{3}\right) \\
= & \frac{1}{2}(m+M)\left(\dot{z}_{1} \dot{z}_{1}+\overline{\dot{z}}_{2} \dot{z}_{2}+\overline{\dot{z}}_{3} \dot{z}_{3}\right)-\frac{M}{4}\left(\bar{z}_{1} \dot{z}_{1}+\overline{\dot{z}}_{2} \dot{z}_{2}\right)-\frac{3}{2} k\left(\bar{z}_{1} z_{1}+\bar{z}_{2} z_{2}\right)
\end{aligned}
$$

and the dissipation function is

$$
\mathscr{F}=\frac{1}{2} \eta\left(\dot{z}_{1} \dot{z}_{1}+\overline{\dot{z}}_{2} \dot{z}_{2}+\overline{\dot{z}}_{3} \dot{z}_{3}\right)
$$

The equations of motion (2) become

$$
\frac{d}{d t} \frac{\partial L}{\partial \overline{\dot{z}}_{i}}-\frac{\partial L}{\partial \bar{z}_{i}}+\frac{\partial \mathscr{F}}{\partial \overline{\dot{z}}_{i}}=0
$$

for $i=1,2,3$. These equations are

$$
\begin{aligned}
(m+M) \ddot{z}_{1}-\frac{M}{2} \ddot{z}_{1}+3 k z_{1}+\eta \dot{z}_{1} & =0, \\
(m+M) \ddot{z}_{2}-\frac{M}{2} \ddot{z}_{2}+3 k z_{2}+\eta \dot{z}_{2} & =0 \\
(m+M) \ddot{z}_{3}+\eta \dot{z}_{2} & =0 .
\end{aligned} \quad \text { and }
$$

The simplified equations take the form

$$
\begin{aligned}
\left(m+\frac{M}{2}\right) \ddot{z}_{1}+\eta \dot{z}_{1}+3 k z_{1} & =0, \\
\left(m+\frac{M}{2}\right) \ddot{z}_{2}+\eta \dot{z}_{2}+3 k z_{2} & =0 \\
(m+M) \ddot{z}_{3}+\eta \dot{z}_{2} & =0 .
\end{aligned}
$$


The natural frequencies of the underdamped harmonic variations in the symmetry coordinates $z_{i}$ may be listed immediately:

$$
f_{1}=f_{2}=\frac{1}{2 \pi} \frac{\sqrt{12 k(m+M / 2)-\eta^{2}}}{2(m+M / 2)}
$$

and $f_{3}=0$.

If the radicand is less than or equal to zero, the vibrations disappear and the return of the symmetry coordinates from their initial to their equilibrium values is described by decaying exponential functions.

\section{The general case for $N$ identical particles}

Let us consider $N$ identical particles symmetrically arranged on a fixed circle with each connected to its neighbors by springs having force constant $k$ and extended mass $M$. Each particle has mass $m$. The frictional force acting on each particle is proportional to its velocity and is given by $-\eta \dot{x}_{i}$. The circular arrangement may be taken to be the result of identifying the first and $(N+1)$-th particles in a linear chain. For large $N$, this identification yields a model of a one-dimensional crystal. The replacement of the linear chain by the circular arrangement is an application of the Born cyclic condition to the chain. A portion of the circular arrangement is indicated in Figure 3.

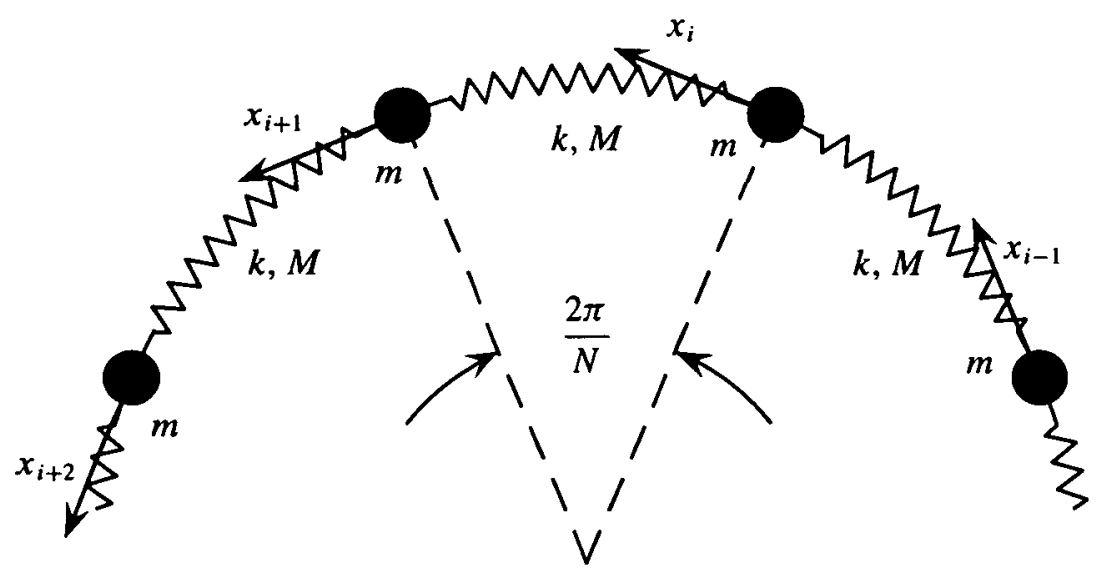

FigURE 3. The circle for large $N$.

Writing the energies, Lagrangian and dissipation function for the general case is every bit as straightforward as for the special case with $N=3$ although the notation becomes extensive. 
The combined kinetic energy of particles and springs is

$$
\frac{1}{2}(m+M) \tilde{\dot{X}} I \dot{X}-\frac{1}{2}\left(\frac{M}{6}\right) \tilde{X} V X,
$$

where $I$ represents the $N$-by- $N$ identity matrix, $V$ is the $N$-by- $N$ matrix

$$
\left(\begin{array}{rrrlrr}
2 & -1 & 0 & \ldots & 0 & -1 \\
-1 & 2 & -1 & \ldots & 0 & 0 \\
0 & -1 & 2 & \ldots & 0 & 0 \\
\vdots & \vdots & \vdots & & \vdots & \vdots \\
-1 & 0 & 0 & \ldots & -1 & 2
\end{array}\right)
$$

$\dot{X}=\operatorname{col}\left(\dot{x}_{1} \dot{x}_{2} \dot{x}_{3} \ldots \dot{x}_{N}\right)$ and $\tilde{\dot{X}}=\left(\dot{x}_{1} \dot{x}_{2} \dot{x}_{3} \ldots \dot{x}_{N}\right)$.

The elastic potential energy may be written as

$$
\frac{1}{2} k \tilde{X} V X
$$

where $X=\operatorname{col}\left(x_{1} x_{2} x_{3} \ldots x_{N}\right)$ and $\widetilde{X}=\left(x_{1} x_{2} x_{3} \ldots x_{N}\right)$. The dissipation function is

$$
\mathscr{F}=\frac{1}{2} \eta \widetilde{X} I \dot{X}
$$

The Lagrangian becomes

$$
L=\frac{1}{2}(m+M) \tilde{\dot{X}} I \dot{X}-\frac{1}{2}\left(\frac{M}{6}\right) \tilde{\dot{X}} V \dot{X}-\frac{1}{2} k \widetilde{X} V X .
$$

The unitary transformation to symmetry coordinates $z_{1}, z_{2}, z_{3}, \ldots, z_{N}$ is accomplished by a matrix similarity transformation with

$$
U=\frac{1}{\sqrt{N}}\left(\begin{array}{ccccc}
\exp (2 \pi i / N) & \exp (4 \pi i / N) & \exp (6 \pi i / N) & \ldots & 1 \\
\exp (4 \pi i / N) & \exp (8 \pi i / N & \exp (12 \pi i / N) & \ldots & 1 \\
\exp (6 \pi i / N) & \exp (12 \pi i / N) & \exp (18 \pi i / N) & \ldots & 1 \\
\vdots & \vdots & \vdots & \ldots & \vdots \\
1 & 1 & 1 & \ldots & 1
\end{array}\right)
$$

and its transposed complex conjugate $U^{-1}$. The matrix $U$ is derived from the irreducible representations of the plane rotations of a regular $N$-sided polygon. 
The transformed Lagrangian is

$$
\begin{aligned}
& L=\frac{1}{2}(m+M) \tilde{\dot{X}} U^{-1} U \dot{X}-\frac{1}{2}\left(\frac{M}{6}\right) \tilde{\dot{X}} U^{-1}\left(U V U^{-1}\right) U \dot{X} \\
& -\frac{1}{2} k \widetilde{X} U^{-1}\left(U V U^{-1}\right) U X=\frac{1}{2}(m+M) \tilde{\dot{Z}} \dot{Z} \\
& -\frac{1}{2}\left(\frac{M}{6}\right) \dot{Z}\left(\begin{array}{cccccc}
4 \sin ^{2} \frac{\pi}{N} & 0 & 0 & \ldots & 0 & 0 \\
0 & 4 \sin ^{2} \frac{2 \pi}{N} & 0 & \ldots & 0 & 0 \\
0 & 0 & 4 \sin ^{2} \frac{3 \pi}{N} & \ldots & 0 & 0 \\
\vdots & \vdots & \vdots & & \vdots & \vdots \\
0 & 0 & 0 & \ldots & 4 \sin ^{2} \frac{(N-1) \pi}{N} & 0 \\
0 & 0 & 0 & \ldots & 0 & 0
\end{array}\right) \dot{Z} \\
& -\frac{1}{2} k \widetilde{Z}\left(\begin{array}{cccccc}
4 \sin ^{2} \frac{\pi}{N} & 0 & 0 & \ldots & 0 & 0 \\
0 & 4 \sin ^{2} \frac{2 \pi}{N} & 0 & \ldots & 0 & 0 \\
0 & 0 & 4 \sin ^{2} \frac{3 \pi}{N} & \ldots & 0 & 0 \\
\vdots & \vdots & \vdots & & \vdots & \vdots \\
0 & 0 & 0 & \ldots & 4 \sin ^{2} \frac{(N-1) \pi}{N} & 0 \\
0 & 0 & 0 & \ldots & 0 & 0
\end{array}\right) Z
\end{aligned}
$$

where $Z=\operatorname{col}\left(z_{1} z_{2} z_{3} \ldots z_{N}\right), \dot{Z}=\operatorname{col}\left(\dot{z}_{1} \dot{z}_{2} \dot{z}_{3} \ldots \dot{z}_{N}\right), \tilde{Z}=\left(\bar{z}_{1} \bar{z}_{2} \bar{z}_{3} \ldots \bar{z}_{N}\right)$ and $\widetilde{\dot{Z}}=$ $\left(\overline{\dot{z}}_{1} \overline{\dot{z}}_{2} \overline{\dot{z}}_{3} \ldots \overline{\dot{z}}_{N}\right)$ and $\bar{z}_{i}, \overline{\dot{z}}_{i}$ represent the complex conjugates of the coordinate $z_{i}$ and velocity $\dot{z}_{i}$.

The dissipation function becomes

$$
\mathscr{F}=\frac{1}{2} \eta \tilde{Z} \dot{Z}
$$

Again, the equations of motion are given by

$$
\frac{d}{d t}\left(\frac{\partial L}{\partial \bar{z}_{i}}\right)-\frac{\partial}{\partial \bar{z}_{i}}+\frac{\partial \mathscr{F}}{\partial \bar{z}_{i}}=0
$$

for $i=1,2,3, \ldots, N$. Thus we have $N$ equations in which coordinates are now completely separated:

$$
\left[m+\left(1-\frac{2}{3} \sin ^{2} \frac{i \pi}{N}\right) M\right] \ddot{z}_{i}+\eta \dot{z}_{i}+\left(4 k \sin ^{2} \frac{i \pi}{N}\right) z_{i}=0 .
$$

The natural frequency associated with the underdamped harmonic vibration of the $i$-th symmetry coordinate $(i=1,2,3, \ldots, N-1)$ is

$$
f_{i}=\frac{1}{2 \pi} \frac{\left[16 k\left(\left(m+\left(1-\frac{2}{3} \sin ^{2} \frac{i \pi}{N}\right)\right) M \sin ^{2} \frac{i \pi}{N}-\eta^{2}\right]^{1 / 2}\right.}{2\left[m+\left(1-\frac{2}{3} \sin ^{2} \frac{i \pi}{N}\right) M\right]} .
$$


Note that to achieve a vibrating motion for the $i$-th symmetry coordinate, $\eta$ must be small enough that the radicand in the expression for $f_{i}$ remains positive. For $i=N$, $f_{N}=0$.

We also note that if $\eta=0$, the natural frequencies become those found in [1].

\section{Acknowledgement}

The authors thank Professor Reuben Farley of Virginia Commonwealth University for reading this manuscript and for his valuable suggestions.

\section{References}

[1] J. N. Boyd and P. N. Raychowdhury, "A one-dimensional crystal with nearest neighbors coupled through their velocities", J. Dynamic Systems, Measurement and Control 103 (1982) 293-296.

[2] J. N. Boyd and P. N. Raychowdhury, "An application of projection operators to a one-dimensional crystal", Bull. of the Institute of Math., Academia Sinica 7 (1979) 133-134.

[3] J. N. Boyd and P. N. Raychowdhury, "Representation theory of finite Abelian groups applied to a linear diatomic crystal", Internat. J. Math. and Math. Sci. 3 (1980) 559-574.

[4] J. N. Boyd and P. N. Raychowdhury, "A group theoretic approach to generalized harmonic vibrations in a one-dimensional lattice", Internat. J. Math. and Math. Sci. 9 (1986) 131-136.

[5] J. N. Boyd and P. N. Raychowdhury, "A double chain of coupled circuits in analogy with mechanical lattices", Internat. J. Math. and Math. Sci. 14 (1991) 403-406.

[6] J. N. Boyd and P. N. Raychowdhury, "A geometrical approach to maximizing a variance", Appl. Math. Modelling 18 (1994) 697-700.

[7] H. Goldstein, Classical Mechanics (Addison-Wesley, Reading, MA, 1965) 21-22.

[8] M. Hamermesh, Group Theory and Its Application to Physical Problems (Addison-Wesley, Reading, MA, 1962).

[9] A. Nussbaum, "Group theory and normal modes", Amer. J. Physics 36 (1968) 529-539. 